\title{
Comparison of CFD numerical approaches for the simulation of accidental gas release in energy applications
}

\author{
Fabrizio Pappalardo ${ }^{1}$, Alberto Moscatello ${ }^{2}$, Anna Chiara Uggenti ${ }^{2}$, Raffaella Gerboni ${ }^{2}$, Andrea \\ Carpignano $^{2}$, Francesco Di Maio ${ }^{1}$, Riccardo Mereu ${ }^{1}$, Enrico Zio ${ }^{1,3}$ \\ ${ }^{1}$ Department of Energy, Politecnico di Milano, via Lambruschini 4, 20156 Milano, Italy. \\ fabrizio.pappalardo@mail.polimi.it,francesco.dimaio@polimi.it,riccardo.mereu@polimi.it, \\ enrico.zio@polimi.it \\ ${ }^{2}$ Department of Energy, Politecnico di Torino, corso Duca degli Abruzzi, 24, 10141 Torino, Italy. \\ alberto.moscatello@polito.it,anna.uggenti@polito.it,raffaella.gerboni@polito.it, \\ andrea.carpignano@polito.it
}

${ }^{3}$ MINES ParisTech, PSL Research University, CRC, Sophia Antipolis, France

Oil \& Gas plants are risk-relevant complex facilities for the presence of toxic, flammable and pressurized fluids. Risk assessment is mandatory to guarantee plant sustainability and compliance with directives. For offshore plants characterized by congested spaces, semi-empirical models for accident consequence simulation often result in risk overestimation. This could be avoided through Computational Fluid Dynamics (CFD), which guarantees more accurate results. Complex phenomena and geometries, however, entail large computational efforts that force limiting the number of simulations to explore the accident scenarios. This calls for new approaches able to model and simulate complex congested geometries in affordable time, while achieving keeping the required accuracy of the results. In this context, a novel CFD model based on ANSYS Fluent, named SBAM (Source Box Accident Model), has been proposed by the research group of the SEADOG lab in Politecnico di Torino with the aim of simulating complex environments with good accuracy and reduced computational cost. In this work, the results provided by the SBAM model on an accidental high pressure flammable gas release in a platform, are compared with those provided by other tools and models available in the market, and widely used in industrial applications, such as FLACS developed by Gexcon US and KFX developed by DNV-GL.

Keywords: Oil and gas offshore platform, Consequence analysis, safety, accidental release, CFD, ANSYS Fluent, FLACS, KFX

\section{Introduction}

Oil \& Gas plants are risk-relevant complex facilities for the presence of hazardous fluids that may be toxic, flammable and pressurized (PatèCornell, 1993 and Necci et al., 2019). Risk assessment is mandatory to guarantee plant sustainability and its compliance with national and international regulatory framework (Vinnem, 2014).

Consequence evaluation is a crucial step of the Quantitative Risk Assessment (QRA) (Casal, 2007 and Vinnem, 2019), which involves the simulation of hundreds of accidental scenarios. The procedure can be time-consuming; methods guaranteeing an optimal compromise between computational time and results accuracy are sought. Whilst the state-of-practice for onshore installations QRA is well defined and entails relying on the empirical methods usage (such as turbulent free-jet models (Chen and Rodi, 1980), gas dispersion models (Davidson, 1967), jet fire models (TNO, 2005 and Zamejc, 2014), explosions models like "TNT" or "TNO MultiEnergy models" (TNO, 2005), vapor cloud explosion models (Baker et al., 1996)), for the QRA of offshore Oil \& Gas facilities, characterized by a complex congested geometry, empirical methods lead to large overestimation of damage areas and to oversizing of protections, that results in corresponding mechanical overload of the offshore structure.

Recently, the SEADOG Lab Group of Politecnico di Torino proposed a novel CFD method, called SBAM (Source Box Accident Model) alternative to empirical methods, aiming at handling the difficulties due the complexity of the offshore installations.

This work compares SBAM, that uses ANSYS Fluent, with two industrial CFD codes, namely FLACS and KFX. FLACS is a code developed by Gexcon US and is the industrial standard for 


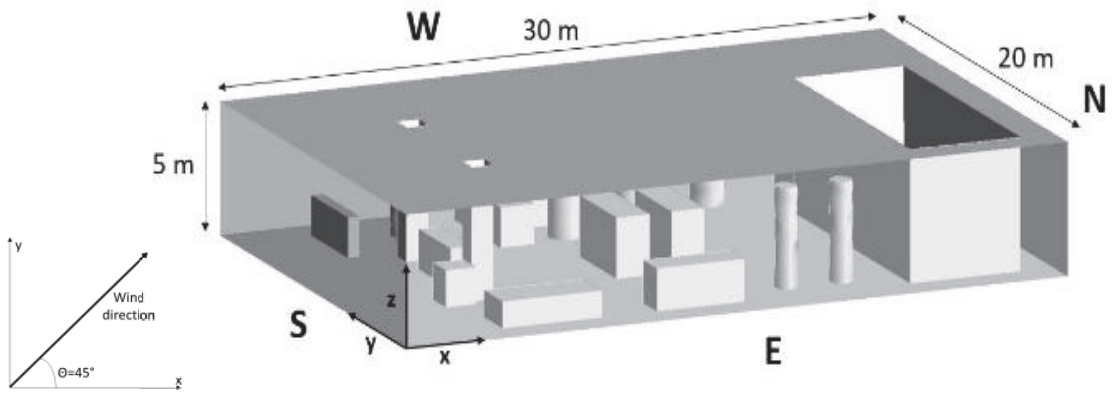

Fig. 1. Production deck CAD

modeling CFD explosion, validated for modelling flammable and toxic releases (Hansen, 2010). Also, it is extensively used in the Oil \& Gas field, and increasingly employed in nuclear industry and other fields involving dust explosion (Gexcon, 2019). KFX is the leading CFD simulator of DNV GL, used to simulate accidents involving the dispersion of hazardous materials, fires and explosions (DNV-GL, 2019).

A benchmark case study is considered, regarding an accident high-pressure methane release in an offshore Oil \& Gas platform. The comparison is carried out considering multiple aspects: software usability, physical and numerical modeling and quantitative results. The benchmark case study is presented in Section 2, whereas Section 3 details the different approaches of the considered tools. Section 4 presents and comments both qualitative and quantitative results of the comparison after the use of the tools.

\section{The benchmark case study}

The analyzed case study concerns an accident high-pressure methane release in an offshore platform deck. This event is considered as a "Major Accident" for this kind of installations (Directive 2013/30EU, 2013). The simulation domain is a simplified 3D CAD version (Fig. 1) of an existing Italian natural gas extraction platform placed in Ravenna Punta Marina, in which all the components (separators, treatment units, storage tanks, etc.) are represented by simple geometrical shapes (cylinders and parallelepipeds). The main characteristic parameters of the accident and their reference values defining a specific accidental scenario are:

- Release pressure: $p_{\text {rel }}=50$ bar;

- Release hole diameter: $d_{\mathrm{e}}=3 \mathrm{~cm}$;

- Release point and direction: $x=3 \mathrm{~m}, \mathrm{y}=2$ $\mathrm{m}, \mathrm{z}=1.5 \mathrm{~m}$ along the $\mathrm{x}$ direction (see Fig. 1);

- Wind direction and intensity: $0.5 x+0.5 y$ (see Fig. 1) and intensity, $u=6 \mathrm{~m} / \mathrm{s}$ (defined according to the reference site of the platform);
- Geometry of the domain (see Fig. 1).

The choice of the representative parameters is made according to (Vivalda et al., 2018), which proposes a method for the identification of a comprehensive set of representative release scenarios for consequences evaluation.

In particular, we consider a high-pressure (7 bars or more) gas release, leading to a highly underexpanded jet (Franquet et al., 2015); when the gas exits, then tends to adjust to the ambient pressure through some expansion-compression waves (shock waves); a Mach disk is formed (Franquet et al., 2015) and supersonic velocity is reached in a region slightly after the release point; therefore, the flow must be treated as compressible during the first "phase", named release phase, whereas, as the gas reaches the ambient pressure and disperses at low velocities $(\sim 10 \mathrm{~m} / \mathrm{s})$ in the remaining portion of the domain, the flow can be treated as incompressible during this "phase" named dispersion. The two "phases" involve different length scales due to the different fluiddynamic natures of the compressible and incompressible parts of the flow. This is even more relevant when we consider as scenario the jet impinging on a cylindrical near-obstacle with diameter $\mathrm{d}_{\mathrm{cyl}}$ equal to $30 \mathrm{~cm}$ and distant $l=30 \mathrm{~cm}$ from the release point is considered. This situation is frequent in the congested offshore installations, in which a free-jet is rather improbable. Moreover, in this way, the capability of the models to reproduce the jet-obstacle interaction is investigated.

\section{Comparison}

The comparison of the different approaches is done with respect to:

- Physical modelling (e.g., governing equations and main assumptions);

- Numerical methods for the solution of governing equations (e.g., turbulence models, mesh configuration, etc.); 


\subsection{Physical modeling approaches}

The basic idea behind the SBAM approach and the FLACS and KFX approach is the same: the release and the dispersion phases are handled separately, using different assumptions and models.

In the SBAM approach, two CFD simulations, through ANSYS Fluent, which solves the NavierStokes equations by a finite volume approach, are realized: one for the release phase and one for the dispersion one. The former, which accounts for the initial expansion of the jet and the interaction with the nearby obstacle, is performed in a dedicated and limited domain called Source-Box (SB) (Carpignano et al., 2017), in which the flow can be assumed and modeled as compressible. The domain is defined large enough to contain all the compressibility effects. The mesh is refined enough to fully resolve the Mach cell and the boundary layer arising from the interaction with the nearby obstacle, therefore all the geometric details have been considered, while the gravitational forces are neglected due to the high inertia of the flow which reaches a supersonic regime. The results of the release simulation are then used as input of the dispersion simulation, which domain is the entire platform deck (Fig. 1), in which the flow is assumed incompressible and gravitational forces become relevant as the velocities are in a subsonic regime (Carpignano et al., 2017). FLACS and KFX use a different, more simplified, approach for the release phase: the initial expansion of the jet is modeled through an empirical method, Birch Model (Birch et al., 1984), and the CFD simulation is only used for the dispersion phase, which is modeled through a Porosity Distributed Resistance (PDR) approach (Patankar and Spalding, 1974). The Birch model and the PDR approach will be briefly summarized in the following paragraphs (3.1.1 and 3.1.2).

\subsubsection{Release phase: Birch model}

In FLACS and KFX the supersonic release of a highly pressurized gas is described according to the semi-empirical Birch model (Birch et al., 1984), obtained by the analysis of compressible sonic jets concentration fields through gas chromatography. This model is applicable in a release pressure range between 2 and 70 bar (Birch et al., 1984). This model evaluates a pseudo-diameter to consider the jet expansion from the release pressure up to the ambient conditions, and in an analogous way a pseudovelocity in correspondence of the pseudodiameter. According to (Birch et al., 1984) the pseudo-diameter $\left(d_{p s}\right)$ can be defined as:

$$
\frac{d_{p s}}{d_{e}}=\sqrt{\frac{p_{r e l}}{p_{a m b}} 0.582 C_{D}}
$$

here $d_{e}$ is the hole diameter, $p_{a m b}$ is the ambient pressure, $C_{D}$ is the discharge coefficient, and $p_{r e l}$ is the pressure upstream the hole section.

The pseudo-velocity $\left(v_{p s}\right)$ is defined as:

$$
v_{p s}=\frac{\dot{m}}{\frac{\pi}{4} d_{p s}^{2} \rho_{g a s, a m b}}
$$

Where $\rho_{\text {gas, amb }}$ is the gas density at ambient conditions and $\dot{m}$ is the gas mass flow rate at hole section.

These pseudo values are imposed on the cell surface corresponding to the new pseudo release point, from where the subsonic dispersion starts.

\subsubsection{Porosity Distributed Resistance}

In FLACS and KFX, the geometry of the dispersion phase is represented as a porous region and the governing equations are obtained using the Porosity Distributed Resistance (PDR) approach (Patankar and Spalding, 1974). This formulation is a generalization of Navier-Stokes (N-S) equations and of Darcy's law, commonly used for flows in porous regions (Miguel et al., 2001). The presence of obstructions modifies the governing equations in two ways: firstly, the obstacles are represented in the control volume so that only the non-blocked areas are available for fluid flow. Secondly, obstacles give additional resistance to the flow, therefore additional drag, turbulence and additional heat transfer effects must be modeled. In FLACS and KFX solvers, all governing equations are written in the following general conservation form:

$$
\begin{gathered}
\frac{\partial}{\partial t}\left(\beta_{v} \rho \phi\right)+\frac{\partial}{\partial x_{i}}\left(\beta_{i} \rho v_{i} \phi\right)=\frac{\partial}{\partial x_{i}}\left(\beta_{i} \rho \Gamma_{\phi} \frac{\partial \phi}{\partial x_{i}}\right)+ \\
\beta_{v}\left(S_{\phi}+R_{\phi}\right)
\end{gathered}
$$

where $\beta_{v}$ is the volume porosity, $\beta_{i}$ is the area porosity in the $i$ direction, $v_{i}$ is the velocity in $i$ direction, $\phi$ is a general variable, $\Gamma_{\phi}$ is the effective turbulence coefficient, $\mathrm{S}_{\phi}$ is the source term for $\phi$, and $\mathrm{R}_{\phi}$ represents additional drag, additional turbulence or additional heat transfer caused by solid obstruction.

This approach permits to model complex geometries such as Oil \& Gas installations or industrial sites, characterized by a wide range of geometry length scales, by defining a suitable grid resolution for larger scales (Cormier et al., 2009), and special sub-grid models for drag and turbulence to account for smaller geometries. 
From Eq. 3, the modified N-S momentum equation could be easily found:

$$
\begin{gathered}
\frac{\partial}{\partial t}\left(\beta \rho v_{i}\right)+\frac{\partial}{\partial x_{j}}\left(\beta_{i} \rho v_{j} v_{i}\right)-\frac{\partial}{\partial x_{j}}\left(\beta_{i} \sigma_{i j}\right)= \\
-\beta \frac{\partial(p)}{\partial x_{i}}+F_{i}+\beta G_{b}
\end{gathered}
$$

where:

$$
F_{i}=-\beta\left(F_{c_{i}}+F_{R_{i}}\left|v_{i}\right|\right) v_{i}
$$

Where $v_{i}$ is the mean velocity vector in the $i$ direction, $\beta$ is the volume porosity, $\beta_{i}$ is the area porosity in the $i$-direction, $v_{j}$ is the mean velocity vector in the $j$-direction, $\sigma_{i j}$ is the stress tensor, $G_{b}$ is the buoyancy. The additional term in the modified N-S momentum equation is a drag, it is modelled through $F_{i}$ which is the flow resistance in the $i$ direction. As shown in Eq. 5, its value can be calculated as a sum of $F_{c x}\left(\mathrm{~kg} / \mathrm{s} / \mathrm{m}^{3}\right)$ and $F_{R x}$ $\left(\mathrm{kg} / \mathrm{m}^{4}\right)$ which are respectively, the resistance constant in the $x$-direction and the speed resistance factor in the $x$-direction.

Obviously, the porosity field varies according to the presence of an obstacle in the Control Volume $(\mathrm{CV})$ : the $\beta_{x}$ coefficients are assumed equal to zero in a CV totally occupied by solid, equal to one if the $\mathrm{CV}$ is not occupied by solid, and values between 0 and 1 if the $\mathrm{CV}$ is partially occupied by solid regions; in this last case, the extraturbulence due to the presence of solid is modeled through a drag factor.

\subsection{Numerical methods comparison}

Numerical methods comparison is performed with respect to:

- Mesh;

- Boundary Conditions (BCs);

- Turbulence models.

\subsubsection{Mesh}

In the SBAM approach, an unstructured tetrahedral mesh is created in Ansys Fluent for both the release and the dispersion simulations as it permits to save setup-time and limit mesh skewness. In fact, as complex geometries are involved both in the release and dispersion phases, the creation of a structured mesh can be extremely time-consuming or not possible, and it could decrease the mesh quality. In addition, the unstructured mesh has a high efficiency mesh distribution, which permits the create fewer cells with respect to the structured one (Ansys Fluent, 2018). In FLACS and KFX only a cartesian structured mesh can be created. Furthermore, the construction of the mesh in FLACS and KFX is limited by the size of the mesh smallest grid cell near the release point; in fact, once the release pseudo-area is defined $\left(\mathrm{A}_{\text {leak }}\right)$, through $\mathrm{d}_{\mathrm{ps}}$ (see Eq. $1)$, the mesh smallest cell area $\left(A_{\text {cell }}\right)$ must satisfy the following relation: $A_{\text {cell }}>A_{\text {leak }}$. Due to this limit, some geometry features of the domain can be lost during the mesh creation: it can happen that objects with a characteristic length of the order of $d_{p s}$ could be lost; neglecting the presence of such small objects can induce significant errors if they occur in high-speed zones since the flow path can be strongly influenced by their presence.

\subsubsection{Boundary conditions}

In the release simulation of the SBAM model, a $\mathrm{CH}_{4}$ concentration equal to 1 and a pressure of 50 bar are imposed on the release hole; the cylindrical nearby obstacle is modeled as a wall with no-slip conditions and ambient pressure is imposed on the external faces of the source box (SB).

Concerning the SBAM dispersion simulation, Fig. 1 can be used as a reference to explain the boundary conditions (BCs): the lateral faces of the deck are named using cardinal points (e.g. the South face is indicated with an S).

The wind velocity is imposed on $\mathrm{S}$ and $\mathrm{E}$ side, in order to reproduce a uniform wind profile of $6 \mathrm{~m} / \mathrm{s}$ impacting on the deck with direction $0.5 x+0.5 y$. $\mathrm{On} \mathrm{N}$ and $\mathrm{W}$ sides, atmospheric pressure is imposed, in fact from these faces the flow is discharged towards the external environment. All the obstacles, roof and floor of the plated deck are modeled as walls with no-slip conditions. The dispersion input values are taken from the release simulation on the boundaries of the SB: these are the velocity and $\mathrm{CH}_{4}$ concentration profiles on the outer faces of the SB that assumes the role of "gas source" for the dispersion simulation.

In FLACS and KFX a unique simulation is performed, i.e. the release and the dispersion parameters are all imposed in the same simulation. At the release point, the release hole size, the type of gas and its thermodynamic conditions are specified; then, FLACS and KFX automatically evaluate the pseudo-source through the Birch model, described in chapter 3.1. A wind uniform profile is imposed on $\mathrm{S}$ and $\mathrm{E}$ sides (analogous to SBAM). On $\mathrm{W}$ and $\mathrm{N}$ sides, ambient pressure is imposed in KFX while a mass flow outlet is used in FLACS; this is suggested in (Gexcon, 2019), in order to obtain a convergent solution.

Due to the PDR approach, in FLACS and KFX it is not possible to model the object surfaces as walls, in fact, a 'mass flow outlet' condition is applied to all the solid objects, then the resistance to flow created by the obstacles is taken in consideration through the porosity value in the 
equation, i.e. the porosity is zero when an object is encountered.

\subsubsection{Turbulence models}

In the SBAM model, both the release and the dispersion simulations are performed in steadystate mode and both the phases use the SST $k-\omega$ model as turbulence closure equation of the N-S equations. Since in the release simulation an under-expanded jet is reproduced (i.e. a transonic behavior is present), the SST $k-\omega$ is used as suggested for this kind of flows in (Ansys Fluent, 2018). Furthermore, the SST $k-\omega$ model has been validated for under-expanded jets by (Novembre et al., 2006; Liu et al., 2014) against the data available in literature (Eggins and Jackson, 1974). The ideal gas law is used to evaluate the density evolution due to the compressible nature of the flow. For the dispersion subsonic flow regime, the SST $k-\omega$ has been validated by (Li et al., 2016), in which a natural gas dispersion in a room was simulated using SST $k-\omega$ model and validated against the experimental data obtained by (Ivings et al., 2010).

The choice to perform a steady-state analysis is justified by the fact that, in real cases, the detection time of the safety sensors is so long that the flammable cloud transient formation and growth cannot be properly detected; it is highly plausible that the alarm signal is given only after the final cloud configuration is reached. Moreover, since many simulations are required for the QRA, the transient simulations would need a computational time not compatible with the design phase of the plant, thus preventing the possibility of a fruitful feedback on the design.

As in Fluent, also in FLACS a steady-state simulation is performed, while KFX permits only transient simulations. Both FLACS and KFX permit to use only the $k-\varepsilon$ turbulence model; this model is widely used in the industrial field and validated for turbulent free flows (Launder and Spalding, 1974); in particular, the Standard $k-\varepsilon$ model is used. The study of (Hang et al., 2013) demonstrated that this model was better than the other $k-\varepsilon$ models to simulate dispersion in an urban environment. Also (Fiates and Vianna, 2016) applied the Standard $k-\varepsilon$ model to simulate jet releases in an industrial setup and reported good predictions of the axial velocity decay. Furthermore, it is interesting to see the mathematical formulation of the equations for $k$ and $\varepsilon$ in the PDR approach, where $k$ is the turbulent kinetic energy and $\varepsilon$ is the rate of dissipation of turbulent kinetic energy (Eq. 6 and Eq. 7).

$$
\frac{\partial}{\partial t}\left(\beta_{v} \rho k\right)+\frac{\partial}{\partial x_{j}}\left(\beta_{j} \rho v_{j} k\right)=
$$

$$
\begin{gathered}
\frac{\partial}{\partial x_{j}}\left(\beta_{j} \frac{\mu_{e f f}}{\sigma_{k}} \frac{\partial k}{\partial x_{j}}\right)+\beta_{v} P_{k}-\beta_{v} \rho \epsilon \\
\frac{\partial}{\partial t}\left(\beta_{v} \rho \epsilon\right)+\frac{\partial}{\partial x_{j}}\left(\beta_{j} \rho v_{j} \epsilon\right)= \\
\frac{\partial}{\partial x_{j}}\left(\beta_{j} \frac{\mu_{e f f}}{\sigma_{\epsilon}} \frac{\partial \epsilon}{\partial x_{j}}\right)+\beta_{v} P_{\epsilon}-\frac{\beta_{v} \rho \epsilon^{2}}{k}
\end{gathered}
$$

Where $\mathrm{v}_{\mathrm{j}}$ is the velocity in the $j$-direction, $\sigma_{\varepsilon}$ and $\sigma_{k}$ are the turbulent Prandtl and Schmidt numbers, $\mu_{\text {eff }}$ is the effective viscosity, $P_{k}$ and $P_{\varepsilon}$ are respectively the production rate of $k$ and production rate of $\varepsilon . P_{k}$ is modeled as a sum of four distinct terms contributing to turbulence generation due to fluid-shear $\left(G_{s}\right)$, wall-shear $\left(G_{w}\right)$, buoyancy $\left(G_{b}\right)$ and sub-grid objects $\left(G_{0}\right)$. The latter term considers the additional turbulence during the interactions between fluid and solid through the defined drag coefficient.

Both the tools use also the ideal gas law to close the set of equations composed by N-S and turbulence equations.

The use of these RANS (Reynolds Averaged Navier-Stokes) models guarantees a good costaccuracy compromise for the consequences' evaluation in a risk assessment; in fact, the estimation of average distributions of the relevant quantities (e.g. flammable gas concentration) is appropriate for the evaluation of the area involved by the accident.

\section{Simulation results}

\subsection{Qualitative comparison}

We consider the simulated volume occupied by the gas cloud within two decks and specifically the portion up to a height $(\mathrm{z})$ of $0.5 \mathrm{~m}$ with a concentration within the flammability limits. This can be assumed as a risk metric for qualitative comparison of the considered approaches, since we assume the probability of ignition is higher in the lower part of the deck, and personnel presence is probable. In particular, we assume the gas to be released is $\mathrm{CH}_{4}$, the flammability limits are: $5 \%$ (Lower Flammability Limit - LFL) and $15 \%$ (Upper Flammability Limit - UFL) by volume of air. Hence, the contours of methane concentration within its flammability range on a section at $\mathrm{z}=$ $0.5 \mathrm{~m}$ are shown in Fig 2, Fig. 3 and Fig. 4 respectively for SBAM, FLACS and KFX.

The shapes of the flammable clouds obtained with FLACS and KFX are similar to each other, while both differ from the cloud obtained with SBAM. The first two appear like a stretched free-jet plume 


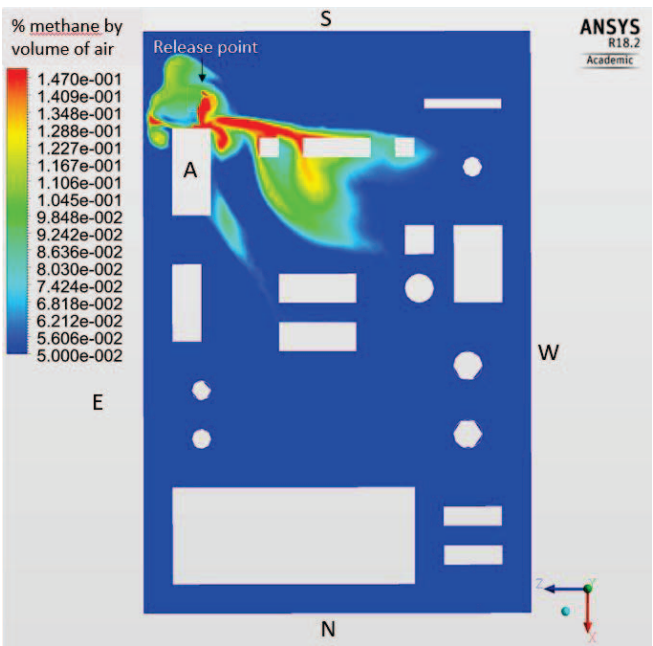

Figure 2 SBAM (Fluent) flammable area in a section at $\mathrm{z}=0.5 \mathrm{~m}$

which is curved due to the wind effect, and slightly influenced by the obstacle presence.

In Fig. 2 (SBAM), it is visible that the jet impacts the obstacle "A" causing a deformation of the cloud; this interaction, not present in the FLACS and KFX cases (Fig. 3 and Fig. 4), strongly influences the evolution of the cloud, generating a highly "irregular" shape of the area involved by the accident.

The difference in the interaction with the objects is mainly due to the fact that FLACS and KFX use the PDR approach; in fact, using the porosity for the domain, the boundary layer is not solved and therefore the modelling of the separation of a flow impacting an object can be affected by this formulation of the problem.

If, on one hand, the PDR approach of FLACS and KFX seems to fail to reproduce the flow-obstacle interactions in a detailed way neglecting many of the geometry features (e.g. curved surfaces are badly approximated), on the other hand, it is much less computationally demanding than SBAM (see section 4.2). A general final qualitative comment can be made on the flexibility of the approaches: this aspect is crucial since it can affect the simulation setup time and the post-processing time during a QRA procedure. FLACS and KFX are specific purpose software, differently from Fluent used for SBAM, which requires a deeper knowledge of the phenomena to be simulated as the implementation of the simulation needs more details in input. Moreover, the creation of the mesh is simpler in KFX and FLACS, since only one type is allowed, and the dimension is almost defined by the release conditions. On the other

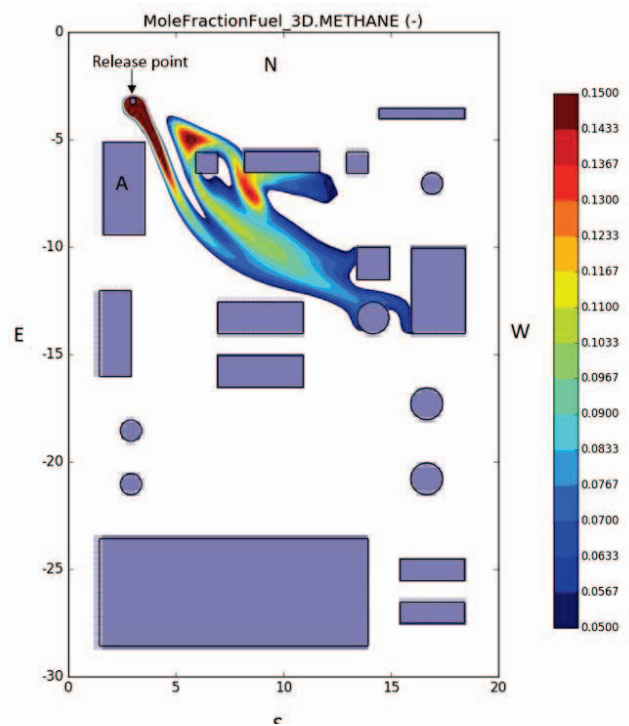

Figure 3 FLACS flammable area in a section at $\mathrm{z}=0.5$ $\mathrm{m}$

hand, in Fluent, the mesh creation is challenging with the advantage that a large flexibility is guaranteed. The post-processing is more flexible and complete in Fluent with respect to the other tools, which do not permit to manage their data through other specific post-processing software, causing sometimes a poor representation of the results.

\subsection{Quantitative comparison}

Table 1 summarizes the results used for quantitative comparison.

Table 1 Results used for quantitative comparison

\begin{tabular}{llll}
\hline & $\begin{array}{l}\text { Total } \\
\text { mass released } \\
(\mathrm{kg})\end{array}$ & $\begin{array}{l}\mathrm{CH}_{4} \\
\text { Flammable } \\
\text { volume } \\
\left(\mathrm{m}^{3}\right)\end{array}$ & $\begin{array}{l}\text { Flammable } \\
\text { mass } \\
(\mathrm{kg})\end{array}$ \\
\hline FLACS & $\sim 16.4$ & $\sim 145$ & $\sim 6.9$ \\
KFX & $\sim 16.2$ & $\sim 184$ & $\sim 8.8$ \\
SBAM & $\sim 14.7$ & $\sim 100$ & $\sim 4.2$ \\
\hline
\end{tabular}

It can be seen that the estimated total mass of $\mathrm{CH}_{4}$ is similar for FLACS, KFX and SBAM, while larger differences arise for flammable volume and mass: in general terms, SBAM gives the lowest estimation of the flammable quantities, while KFX seems to give the most conservative results in terms of physical consequences. Reminding that the total mass of $\mathrm{CH}_{4}$ involved is almost the same, the differences in the flammable volume and mass can be explained considering that in FLACS and KFX a better $\mathrm{CH}_{4}$-air mixing occurs, 


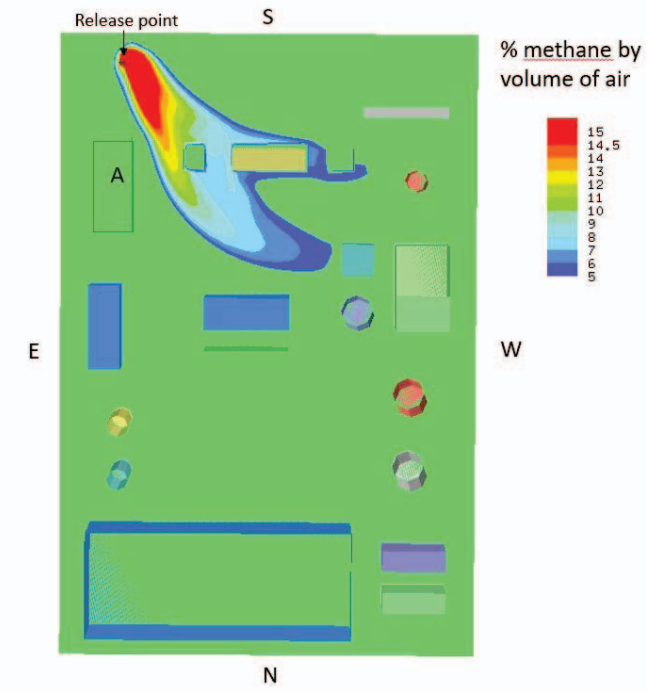

Figure 4 KFX flammable area in a section at $\mathrm{z}=0.5 \mathrm{~m}$

while in SBAM the gas seems to be congested and trapped near the release point; the outcome is that the volume occupied by a sufficiently dispersed mixture (methane concentration between LFL and UFL) is lower.

From a computational time point of view, FLACS results to be the more performant tool as it takes 50 minutes for the simulation (steady-state) on a Dell Tower 7820, while KFX takes 3.5 hours (transient) on the same machine and SBAM takes $7 \mathrm{~h}$ (steady-state) on a Dell Tower 7810.

\section{Conclusions and future works}

In this work, three different tools are compared with respect to their capability of simulating a high-pressure methane release in a platform deck: FLACS, KFX and SBAM.

FLACS and KFX use a CFD-PDR approach combined with a semi-empirical model to describe the entire phenomenon while SBAM is developed in ANSYS Fluent and is purely CFD based.

The main differences in terms of physical modeling, numerical methods, usability, qualitative and quantitative results have been analyzed. The PDR approach used by FLACS and KFX seems to have some criticalities in modeling complex geometries, but the calculation is faster than SBAM, which employs more computational and implementation time but provides more accurate results. Different physical areas impacted by the accident are obtained in the three tools; although some general considerations are possible, a comparison of the flammable areas shapes is not enough to draw conclusions and some risk-oriented indices must be employed for a more significant comparison of the results.

The quantitative comparison is made considering a global quantity (the total methane mass released) and two risk-oriented quantities: the flammable volume and the flammable mass. In this case SBAM calculates smaller flammable quantities than to the other tools, whereas the total mass is found to be in good agreement with the others. The comparison shows that faster software lead to more conservative results.For example, KFX estimates $\sim 100 \%$ more flammable mass than SBAM, which means that the energies and damage to be considered are very different in case of fire and explosion. These differences can be mainly associated to the different $\mathrm{N}-\mathrm{S}$ equations formulation. In fact, the faster tools analyze roughly the supersonic release area, especially in presence of obstacles near the release point; therefore, they might misrepresent the areas involved by fire/explosion accident scenarios. However, it is difficult to assess which result is more realistic since no experimental data are available. For this reason, with regard to SBAM, an experimental campaign is being developed, in order to obtain experimental data with the aim of a code validation. Moreover, CFD numerical results are affected by several uncertainties due to both modeling errors (boundary conditions, initial conditions, geometry, fluid properties, turbulence modeling errors) and numerical errors (discretization, round-off and iterative convergence errors); therefore, a further development of the analysis could concern an uncertainty analysis, in order to allow a more comprehensive results evaluation.

\section{Acknowledgement}

The research presented in this paper has been sponsored by the Italian Ministry of Economic Development's Directorate General for Safety National Mining Office for Hydrocarbons and Georesources.

\section{References}

ANSYS Fluent 18.2 User's Guide, 2018.

Baker, Q., M. Tang, E. Scheier and G. Silva (1996). Vapour Cloud Explosion Analysis. Process Safety Progress Vol. 15, pp. 106-109.

Birch, A. D., D. R. Brown, M. G. Dodson and F. Swaffield (1984). The Structure and Concentration Decay of High Pressure Jets of Natural Gas. Combustion Science and Technology Vol. 36, pp. 249-261.

Carpignano A., T. Corti, A. C. Uggenti, R. Gerboni (2017). Modelling of a supersonic accidental release in Oil\&Gas offshore: characterisation of a 
source box. GEAM Geoingegneria Ambientale Vol. 152:3, pp. 58-64.

Casal, J. (2007). Evaluation of the effects and consequences of Major Accidents in industrial plants-Chapter 11. Elsevier.

Chen, C.J. and W. Rodi (1980). Vertical turbulent buoyant jets. Pergamon Press.

Cormier, B. R., R. Qi, G. Yun, Y. Zhang, M. S. Mannan (2009). Application of computational fluid dynamics for LNG vapor dispersion modeling: A study of key parameters. Journal of Loss Prevention in the Process Industries Vol. 22, pp. 332-352.

Davidson, G. (1967). Simultaneous trajectory and dilution predictions from a simple integral plume model. Atmospheric Environment Vol. 23, pp. 341349.

DNV-GL (2019) KFX-EXISM V.3.4 User Manual, Technical manual.

European Union (2013). Directive 2013/30EU of the European Parliament and of the Council of 12 June 2013.

Eggins, P. L. and D. A. Jackson (1974). Laser-Doppler velocity measurements in an under-expanded free jet. Journal of Physics D: Applied Physics Vol. 7, Number 14.

Fiates J. and S. S. V. Vianna (2016) Numerical modelling of gas dispersion using OpenFOAM. Process Safety and Environmental Protection. Vol. 74, pp. 277293.

Franquet, E., V.Perrier, S. Gibout and P. Bruel (2015). Free underexpanded jets in a quiescent medium: A review. Progress in Aerospace Science Vol. 77, pp. 25-53.

Gexcon, A.S. (2019). Flacs v10.4r2 user's manual, Technical report.

Hang J., Z. Luo, M. Sandberg and J. Gong (2013). Natural ventilation assessment in typical open and semi-open urban environments under various wind directions. Building and Environment Vol. 70, pp. 318-333.

Hansen O.R, F. Gavelli, M. Ichard and S.G. Davids (2010). Validation of FLACS against experimental data sets from the model evaluation database for LNG vapor dispersion. Journal of Loss Prevention in the Process Industries 23, pp. 857-877.

Ivings, M. J., S. E. Gant, C. J. Saunders and D. J. Pocock (2010). Flammable gas cloud build up in a ventilated enclosure. Journal of Hazardous Materials Vol 184 (1), pp. 170-176.

Launder B. E. and D.B. Spalding (1974). The numerical computation of turbulent flows. Computer Methods in Applied Mechanics and Engineering Vol. 3 (2), pp. 269-289.

Li, X. J., R. P. Zhou, and D. Konovessis (2016). CFD analysis of natural gas dispersion in engine room space based on multi-factor coupling. Ocean Engineering Vol. 111, pp. 524-532.

Liu, X., A. Godbole, C. Lu, G. Michal and P. Venton, (2014). Source strength and dispersion of $\mathrm{CO}_{2}$ releases from high-pressure pipelines: CFD model using real gas equation of state. Applied Energy Vol. 126, pp. 56-68.

Miguel, A. F., N. J. van de Braak, A. M. Silva and G. P. A. Bot (2001). Wind-induced airflow through permeable materials. Journal of Wind Engineering and Industrial Aerodynamics Vol. 89, pp. 59-72.

Necci, M., S. Tarantola, B. Vamanu, E. Krausmann and L. Ponte (2019). Lessons learned from offshore oil and gas incidents in the Arctic and other ice-prone seas. Ocean Engineering Vol. 185, pp. 12-26.

Novembre, N., F. Podenzani and E. Colombo (2006). Numerical study for accidental gas releases from high pressure pipelines. Proceedings of the European Conference on Computational Fluid Dynamics.

Patankar, S. V. and D. B. Spalding (1974). A calculation procedure for the transient and steady-state behaviour of shell-and-tube heat exchangers. In N. H. Afgan, \& E. V. Schundler (Eds.), Heat exchangers: design and theory sourcebook (pp. 155-176). McGraw-Hill.

Paté-Cornell, M. (1993). Risk analysis and risk management for offshore platforms: Lessons from the Piper Alpha accident. Journal of Offshore Mechanics and Arctic Engineering 115, pp. 179190.

TNO, (2005). Yellow Book - Methods for the Calculation of Physical Effects due to releases of hazardous materials (Liquids and Gases). Springer.

Vinnem, J.E. (2014). Offshore Risk Assessment Vol. 1 (Third Edition). Springer.

Vinnem, J.E. (2019). Offshore Risk Assessment vol 2 Chapter 14 (Fourth Edition). Springer.

Vivalda, C., R. Gerboni and A. Carpignano (2018). A practical approach to risk-based gas monitoring system design for oil and gas offshore platforms. Proceedings of the $14^{\text {th }}$ Probabilistic Safety Assessment and Management Conference.

Zamejc, E. (2014). API Standard 521 new alternative method to evaluate fire relief for pressure relief device sizing and depressuring system design. Journal of Loss Prevention in the Process Industries Vol. 27, pp. 21-31. 